\title{
Heavy Hybrids in QCD effective theories
}

\author{
Nora Brambilla ${ }^{\text {a* }}$ \\ ${ }^{a}$ Institut für Theoretische Physik, Boltzmanngasse 5, \\ A-1090 Vienna, Austria
}

\begin{abstract}
We present model independent predictions on the short range behavior of the energies of the gluonic excitations between static quarks (hybrid potentials) in an effective field theory framework (pNRQCD).
\end{abstract}

\section{INTRODUCTION}

One of the clearest way of studying the heavy hybrid systems is from the energies of the gluonic excitations between static quarks, the so called excited gluonic potentials $V_{H}$. These energies are calculated on the lattice using Wilson loops with insertions of operators of a definite symmetry (see below) in the end point strings. The frame is supplied by the Born-Oppenheimer approximation. In this picture the quarks and the gluons are respectively slow and fast degrees of freedom and in the limit $\Lambda_{\mathrm{QCD}} / m \rightarrow \infty,(m=$ mass of the quark), the static gluonic energies $V_{H}$ are treated as adiabatic potentials for the $Q \bar{Q}$ pair and inserted in the Schrödinger equation to get the masses of the hybrids. Of course, the shape of $V_{H}$ as a function of the $Q \bar{Q}$ distance $r$ is an interesting observable of QCD.

Here, we show how the appropriate QCD effective field theory description of the heavy quark bound systems can provide us with model independent predictions on the short range behavior of $V_{H}$.

\section{2. pNRQCD}

The existence of a hierarchy of energy scales $m \gg m v \gg m v^{2}, v \ll 1$ being the quark velocity, in the heavy quark non-relativistic bound systems allows us to substitute scale by scale QCD with simpler but equivalent effective theories. Integrating out the hard scale $m$ one passes from

\footnotetext{
${ }^{*}$ Marie Curie fellowship, Contract No. ERBFMBICT961714; 'Acciones Integradas' 1999-2000, project No. 13/99.
}

QCD to NonRelativisticQCD (NRQCD), while integrating out the soft scale $m v$ one passes from NRQCD to potential NRQCD (pNRQCD) [1]. In this last effective theory only ultrasoft (US=with energy much smaller than $m v$ ) degrees of freedom remain dynamical. The surviving fields are the $Q-\bar{Q}$ states (with US energy) and the US gluons. The $Q-\bar{Q}$ states can be decomposed into a singlet $(\mathrm{S})$ and an octet $(\mathrm{O})$ under color transformation. The relative coordinate $\mathbf{r}=\mathbf{x}_{1}-\mathbf{x}_{2}$, whose typical size is the inverse of the soft scale, is explicit and can be considered as small with respect to the remaining (US) dynamical lengths in the system. Hence the gluon fields can be systematically expanded in $\mathbf{r}$ (multipole expansion). Therefore the pNRQCD Lagrangian is constructed not only order by order in $1 / m$, but also order by order in r. As a typical feature of an effective theory, all the non-analytic behavior in $\mathbf{r}$ is encoded in the matching coefficients, which can be interpreted as potential-like terms. The pNRQCD Lagrangian density is given at the next to leading order in the multipole expansion by:

$$
\begin{gathered}
\mathcal{L}_{\mathrm{pNRQCD}}=\operatorname{Tr}\left\{\mathrm{S}^{\dagger}\left(i \partial_{0}-\frac{\mathbf{p}^{2}}{m}-V_{s}(r)+\ldots\right) \mathrm{S}\right. \\
\left.+\mathrm{O}^{\dagger}\left(i D_{0}-\frac{\mathbf{p}^{2}}{m}-V_{o}(r)+\ldots\right) \mathrm{O}\right\} \\
+g V_{A}(r) \operatorname{Tr}\left\{\mathrm{O}^{\dagger} \mathbf{r} \cdot \mathbf{E} \mathrm{S}+\mathrm{S}^{\dagger} \mathbf{r} \cdot \mathbf{E} \mathrm{O}\right\} \\
+g \frac{V_{B}(r)}{2} \operatorname{Tr}\left\{\mathrm{O}^{\dagger} \mathbf{r} \cdot \mathbf{E} \mathrm{O}+\mathrm{O}^{\dagger} \mathrm{Or} \cdot \mathbf{E}\right\}
\end{gathered}
$$

where $\mathbf{R} \equiv\left(\mathbf{x}_{1}+\mathbf{x}_{2}\right) / 2, \mathrm{~S}=\mathrm{S}(\mathbf{r}, \mathbf{R}, t)$ and $\mathrm{O}=$ $\mathrm{O}(\mathbf{r}, \mathbf{R}, t)$ are the singlet and octet wave functions respectively. All the gauge fields in Eq. (11) are 
evaluated in $\mathbf{R}$ and $t$. In particular $\mathbf{E} \equiv \mathbf{E}(\mathbf{R}, t)$ and $i D_{0} \mathrm{O} \equiv i \partial_{0} \mathrm{O}-g\left[A_{0}(\mathbf{R}, t), \mathrm{O}\right] . V_{s}$ and $V_{o}$ are the singlet and octet matching potentials; $V_{A}$ and $V_{B}$ are matching coefficients.

In QCD another physically relevant scale has to be considered, i.e $\Lambda_{Q C D}$, the scale at which the nonperturbative effects become relevant. As far as $m v \gg \Lambda_{Q C D}$, the matching between NRQCD and pNRQCD can be performed perturbatively.

We believe that pNRQCD is the most appropriate effective theory to describe the physics of heavy quark bound systems. For details see [1].

\section{GLUELUMPS in pNRQCD[间]}

Although pNRQCD is originally designed to study $Q-\bar{Q}$ systems of large but finite mass, it is interesting to study its static limit. We shall restrict ourselves to the case of pure gluodynamics. We call gluelump the adjoint source in presence of a gluonic field

$H(\mathbf{R}, \mathbf{r}, t)=H^{a}(\mathbf{R}, t) O^{a}(\mathbf{R}, \mathbf{r}, t)$.

In the static limit there are no space derivatives in the Lagrangian, and hence $\mathbf{r}$ and $\mathbf{R}$ are good quantum numbers. The spectrum then consists of static energies which depend on $\mathbf{r}$ (translation invariance forbids $\mathbf{R}$ dependences) and on the only other scale in this problem, $\Lambda_{\mathrm{QCD}}$.

In the limit $\Lambda_{\mathrm{QCD}} \ll 1 / r$, the spectrum of the theory can be read from the Lagrangian (11). In particular, the leading order solution corresponds to the zeroth order of the multipole expansion. At this order the dynamics of the singlet and octet fields decouple. Hence, the gluonic excitations between static quarks in the short-distance limit correspond to the gluelumps. Depending on the glue operator $H$ and its symmetries, the gluelump operator (2) describes a specific gluonic excitation between static quarks and its static energy $V_{H}$.

In NRQCD (as in QCD) the gluonic excitations between static quarks have the same symmetries of a diatomic molecule plus charge conjugation. In the centre-of-mass system these correspond to the symmetry group $D_{\infty h}$ (substituting the parity generator by $\mathrm{CP}$ ). According to it the mass eigenstates are classified in terms of the angular momentum along the quark-antiquark axes
$\left(\left|L_{z}\right|=0,1,2, \ldots\right.$ to which one gives the traditional names $\Sigma, \Pi, \Delta, \ldots$ ), CP (even, $g$, or odd, $u$ ), and the reflection properties with respect to a plane passing through the quark-antiquark axes (even, + , or odd, - ). Only the $\Sigma$ states are not degenerate with respect to the reflection symmetry. In Fig. 1 we report the lattice measurement of $V_{H}$ obtained considering Wilson loops with operators of the appropriate symmetry inserted at the end points [2, 迎.

In pNRQCD at lowest order in the multipole expansion, besides the already mentioned symmetries, extra symmetries for the gluonic excitation between static quarks appear. The glue dynamics no longer involves the relative coordinate $\mathbf{r}$. Therefore, the glue associated with a gluonic excitation between static quarks acquires a spherical symmetry. In the centre-of-mass system gluonic excitations between static quarks are, therefore, classified according to representations of $O(3) \times$ $\mathrm{C}$, which we summarize by $L$, the angular momentum, CP and reflection with respect a plane passing through the quark-antiquark axes. Since this group is larger than that one of NRQCD, several gluonic excitations between static quarks are expected to be approximately degenerate in pNRQCD, i.e. in the short-distance limit $r \ll$ $1 / \Lambda_{\mathrm{QCD}}$. We illustrate this point by building up all operators, $H$, up to dimension 3 and by classifying them according to their quantum numbers in NRQCD and pNRQCD in Tab. 1. In Tab. 11 all the operators are intended evaluated in the centre-of-mass coordinates. $\Sigma_{g}^{+}$is not displayed since it corresponds to the singlet state. The prime indicates excited states of the same quantum numbers. The chosen operators for the $\Pi$ and $\Delta$ states are not eigenstates of the reflection operator. This is not important since these states are degenerate with respect to this symmetry. The operators $\mathbf{E}$ and $\mathbf{D} \times \mathbf{B}(\mathbf{B}$ and $\mathbf{D} \times \mathbf{E})$ have the same quantum numbers and are related by the equations of motion so that they project over the same states. From the results of Tab. 11 the following degeneracies are expected in the short-distance limit:

$$
\begin{array}{ll}
\Sigma_{g}^{+\prime} \sim \Pi_{g} ; & \Sigma_{g}^{-} \sim \Pi_{g}^{\prime} \sim \Delta_{g} \\
\Sigma_{u}^{-} \sim \Pi_{u} ; & \Sigma_{u}^{+} \sim \Pi_{u}^{\prime} \sim \Delta_{u} .
\end{array}
$$


Table 1

Matching operators $H$ for the $\Sigma, \Pi$ and $\Delta$ gluonic excitations between static quarks in pNRQCD up to dimensions 3. The covariant derivative is understood in the adjoint representation. $\mathbf{D} \cdot \mathbf{B}$ and $\mathbf{D} \cdot \mathbf{E}$ do not appear, the first because it is identically zero after using the Jacobi identity, while the second gives vanishing contributions after using the equations of motion.

\begin{tabular}{lcr}
\hline $\begin{array}{l}\text { Gluelumps } \\
\left(\operatorname{Tr}\left\{\mathrm{O}_{\mathrm{a}} H^{a}\right\}\right)\end{array}$ & $L=1$ \\
\hline $\begin{array}{l}\Sigma_{g}^{+\prime} \\
\Sigma_{g}^{-}\end{array}$ & $\mathbf{r} \cdot \mathbf{E}, \mathbf{r} \cdot(\mathbf{D} \times \mathbf{B})$ & $L=2$ \\
\hline$\Pi_{g}$ & $\mathbf{r} \times \mathbf{E}, \mathbf{r} \times(\mathbf{D} \times \mathbf{B})$ & $(\mathbf{r} \cdot \mathbf{D})(\mathbf{r} \cdot \mathbf{B})$ \\
\hline$\Pi_{g}^{\prime}$ & & $\mathbf{r} \times((\mathbf{r} \cdot \mathbf{D}) \mathbf{B}+\mathbf{D}(\mathbf{r} \cdot \mathbf{B}))$ \\
\hline$\Delta_{g}$ & & $(\mathbf{r} \times \mathbf{D})^{i}(\mathbf{r} \times \mathbf{B})^{j}+(\mathbf{r} \times \mathbf{D})^{j}(\mathbf{r} \times \mathbf{B})^{i}$ \\
\hline$\overline{\Sigma_{u}^{+}}$ & $\mathbf{r} \cdot \mathbf{B}, \mathbf{r} \cdot(\mathbf{D} \times \mathbf{E})$ & \\
\hline$\Sigma_{u}^{-}$ & $\mathbf{r} \times \mathbf{B}, \mathbf{r} \times(\mathbf{D} \times \mathbf{E})(\mathbf{r} \cdot \mathbf{E})$ \\
\hline$\Pi_{u}$ & & $\mathbf{r} \times((\mathbf{r} \cdot \mathbf{D}) \mathbf{E}+\mathbf{D}(\mathbf{r} \cdot \mathbf{E}))$ \\
\hline$\Pi_{u}^{\prime}$ & & $(\mathbf{r} \times \mathbf{D})^{i}(\mathbf{r} \times \mathbf{E})^{j}+(\mathbf{r} \times \mathbf{D})^{j}(\mathbf{r} \times \mathbf{E})^{i}$ \\
\hline$\Delta_{u}$ &
\end{tabular}

Similar observations have also been made in [3]. In pNRQCD they emerge in a quite clear and straightforward way. Moreover, here we can write explicitly the relevant operators.

So far we have just used the symmetries of $\mathrm{pN}-$ RQCD at lowest order in the multipole expansion. In fact we can go beyond that and predict the shape of the static energies by actually studying the correlators

$$
\begin{gathered}
\left\langle 0\left|H(\mathbf{R}, \mathbf{r}, T / 2) H^{\dagger}\left(\mathbf{R}^{\prime}, \mathbf{r}^{\prime},-T / 2\right)\right| 0\right\rangle \sim \\
\delta^{3}\left(\mathbf{R}-\mathbf{R}^{\prime}\right) \delta^{3}\left(\mathbf{r}-\mathbf{r}^{\prime}\right) e^{-i T V_{H}(r)}
\end{gathered}
$$

for large $T$. At leading order in the multipole expansion we obtain

$$
\begin{aligned}
& V_{H}(r)=V_{o}(r)+ \\
& \left.+\frac{i}{T} \ln \left\langle H^{a}(T / 2) \phi(T / 2,-T / 2)_{a b}^{\mathrm{adj}} H^{b}(-T / 2)\right)\right\rangle,
\end{aligned}
$$

where the $T \rightarrow \infty$ limit is understood. The general structure of the gluonic correlator is the following (the contribution from the continuum is included in the dots)

$$
\begin{aligned}
& \left\langle H^{a}(T / 2) \phi(T / 2,-T / 2)_{a b}^{\mathrm{adj}} H^{b}(-T / 2)\right\rangle^{\text {nonpert. }} \\
& \simeq h e^{-i \Lambda_{H} T}+h^{\prime} e^{-i \Lambda_{H}^{\prime} T}+\ldots
\end{aligned}
$$

Since we are in the static limit, $1 / T \ll \Lambda_{\mathrm{QCD}} \sim$ $\Lambda_{\mathrm{H}}<\Lambda_{\mathrm{H}}^{\prime}<\ldots$, one can approximate the righthand side of Eq. (5) for $T \rightarrow \infty$ by just keeping the first exponential of Eq. (6). Then we get at leading order in the multipole expansion

$V_{H}(r)=V_{o}(r)+\Lambda_{H}$.

Formula (7) states that at leading order in the multipole expansion the short-distance behavior of the static energies for the gluonic excitations between static quarks is described by the perturbative octet potential plus a nonperturbative constant. The constant $\Lambda_{H}$ depends in general on the particular operator $H$, i.e. on the particular gluonic excitation between static quarks. $\Lambda_{H}$ is the same for operators identifying states which are degenerate. Notice also that Eq. (7) can be systematically improved by calculating higher orders in the multipole expansion. In particular, one can look at how the $O(3) \times \mathrm{C}$ symmetry is softly broken to $D_{\infty h}$ in the short-distance limit. Let us compare our results against the best available lattice data (see Fig. 1 and [2]). First of all we observe a tendency of all the static energies except $\Pi_{g}$ to go up at short-distances which may be interpreted as an indication that they want to follow the octet potential shape as required by (7). 


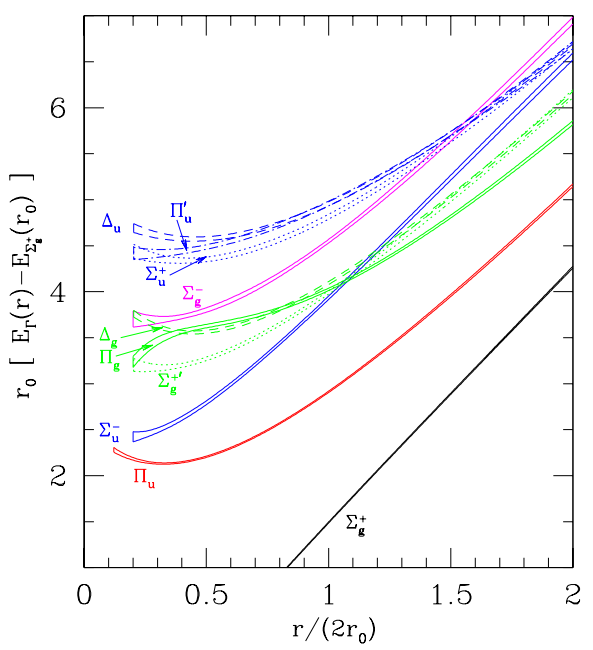

Figure 1. Energies $V_{H}$ for different gluonic excitation between static quarks at distance $r$ from the quenched lattice measurements of [2], $r_{0} \simeq 0.5$ $\mathrm{fm}$. The picture is taken from [1].

The $\Pi_{u}-\Sigma_{u}^{-}$and $\Delta_{u}-\Pi_{u}^{\prime}-\Sigma_{u}^{+}$static energies also show a strong tendency to form a degenerate doublet and triplet respectively at short-distances as precisely predicted in (3). However, something strange is observed for the $g$ static energies. According to (3) a degenerate doublet and triplet should be observed. We can clearly see the doublet $\Pi_{g}-\Sigma_{g}^{+\prime}$ but, as mentioned before, the shape of $\Pi_{g}$ is not compatible with the octet potential. On the other hand $\Delta_{g}-\Sigma_{g}^{-}$miss a state $\Pi_{g}^{\prime}$ to complete the triplet. We suspect that the plotted $\Pi_{g}$ is a superposition of the real $\Pi_{g}$ in the doublet and the $\Pi_{g}^{\prime}$ in the triplet. This would explain its peculiar behavior at small $r$. We expect that future lattice simulations will be able to disentangle these two states and confirm our predictions. It is interesting to notice that also the hierarchy of the states, as displayed in Fig. 1, is reflected in the dimensionality of the operators of Table 1. Lower lying states are characterized by lower dimensional operators if we use a minimal bases with no electric fields. In particular this allows to understand on simple dimensional grounds the highly non-trivial fact that the doublet and triplet static energies lay close together for the $g$ states but quite far apart for the $u$ ones.

The results given above allow us to relate, in the short-distance limit, the behavior of the en- ergies for the gluonic excitation between static quarks with the large time behavior of some gluonic correlators, in particular with their correlation length. It is particularly appealing that we can extract results for the gauge invariant two-point correlator for the gluon field strength tensor: $\left\langle 0\left|F_{\mu \nu}^{a}(t) \phi(t, 0)_{a b}^{\mathrm{adj}} F_{\mu \nu}^{b}(0)\right| 0\right\rangle, \phi$ being the adjoint string. One can parameterize this correlator as a function of two scalar functions: $\left\langle 0\left|\mathbf{E}^{a}(t) \phi(t, 0)_{a b}^{\text {adj }} \mathbf{E}^{b}(0)\right| 0\right\rangle$ and $\left\langle 0\left|\mathbf{B}^{a}(t) \phi(t, 0)_{a b}^{\text {adj }} \mathbf{B}^{b}(0)\right| 0\right\rangle$ with correlations lengths: $T_{E}=1 / \Lambda_{E}$ and $T_{B}=1 / \Lambda_{B}$ respectively. From the results of Ref. [2] displayed in Fig. 1 we can conclude that

$T_{E}<T_{B}$.

So far lattice simulations of the gauge invariant two-point correlator for the gluon field strength tensor have not reached enough precision to confirm this behavior [5]. This would be a nice crosscheck of lattice simulations. On the other hand, recently, a sum rule calculation [6] found evidence in favor of Eq. (8) and also a lattice computation of the gluelump masses [3] seem to confirm Eq. (8). It would be highly desirable to have more precise lattice data in the short-distance limit in order to test our results more quantitatively.

\section{Acknowledgements}

I thank A. Pineda, J. Soto and A. Vairo for the collaboration which produced the results contained in the present paper.

\section{REFERENCES}

1. N. Brambilla, A. Pineda, J. Soto, A. Vairo, hep-ph/9907240; hep-ph/9903355; A. Pineda and J. Soto, Nucl. Phys. B (Proc. Suppl.) 64, (1998) 428.

2. K. J. Juge, J. Kuti and C. Morningstar, heplat/9809015.

3. M. Foster and C. Michael, Phys. Rev. D59 (1999) 094509.

4. C. Michael, hep-ph/9809211.

5. G. Bali, N. Brambilla and A. Vairo, Phys. Lett. B421 (1998) 265 and refs. therein.

6. H. G. Dosch, M. Eidemüller and M. Jamin, Phys. Lett. 452 (1999) 379. 